\title{
N95- 18987
}

1994

30910

WASA/AGEB SUMUER FACULTY FELLOUSHIP PROGRAY

MARSHALI SPACE FLIGHT CENTER

THE UNIVERSITY OF ALABAYA

A PRELIMIMARY AMALYIS OF IOW FREQUEMCY

PRESBURE OSCILIATIONS IN HYBRID ROCKET YOTORS

Prepared By:

Academic Rank:

Institution and Department:
Rhonald M. Jenkins, Ph.D.

Associate Professor

Auburn University

Department of Aerospace Engineering

NASA/MSFC

Laboratory:

Division:

Branch:

Propulsion

Motor systems

Solid Motor Design

MSFC Colleague:

Jerry R. Cook

$\mathrm{XX}$ 


\section{IHWRODUCHIOY}

Past research with hybrid rockets has suggested that certain motor operating conditions are conducive to the formation of pressure oscillations, or flow instabilities, within the motor combustion chamber. These combustionrelated vibrations or pressure oscillations may be encountered in virtually any type of rocket motor and typically fall into three frequency ranges: low frequency oscillations (0-400 Hz); intermediate frequency oscillations (400-1000 Hz); and high frequency oscillations ( $1000 \mathrm{~Hz})^{1}$. In general, combustion instability is characterized by organized pressure oscillations occurring at well-defined intervals with pressure peaks that may maintain themselves, grow, or die out. Usually, such peaks exceed $\pm 5 \%$ of the mean chamber pressure.

For hybrid motors, these oscillations have been observed to grow to a limiting amplitude which may be dependent on factors such as fuel characteristics, oxidizer injector characteristics, average chamber pressure, oxidizer, mass flux, combustion chamber characteristic length $I^{*}$, and grain geometry ${ }^{1}$ Mechanisms which have been postulated as causes of instability in hybrid motors include

Instabilities associated with the time lag of vaporization and combustion of liquid droplets ${ }^{2,3}$. Inadequate droplet atomization and vaporization, as well as inadequate vaporization chamber residence time, are regarded as primary contributing factors. These are, in turn, infíuenced by factors such as droplet size and initial injection velocity $i . e$, , injector design), and vaporization chamber geometry (e.g., volume, the use of splashblocks, etc.). This type of instability is characterized by low frequency pressure oscillations $(5-10 \mathrm{~Hz})$ and it operates generally in the low oxidizer mass flow - low chamber pressure regime ${ }^{4}$.

Instabilities associated with periodic accumulation and break-off of melted layers or char layers from the burning propellant surface. Propellant formulation and thermal response time are believed to be primary contributing factors. This type of instability is characterized by low frequency pressure oscillations (1-100 Hz) and occurs with low regression rate motor operation ${ }^{4}$.

Instabilities associated with some boundary layer mechanism which couples the combustion response (regression rate) to externally imposed pressure oscillations ${ }^{2}$. These instabilities occur in low total mass flux regimes (where radiation is the dominant heat transfer mechanism) and in high total mass flux and/or low chamber pressure regimes (where finite-rate reaction kinetics becomes important) ${ }^{5}$.

Instabilities associated with low impedance LoX feed system design, wherein chamber pressure fluctuations result in Lox flow fluctuations. Generally, it is believed that fluctuations in LoX flow rate may enhance chamber pressure oscillations, but do not initiate them ${ }^{3}$.

Instabilities associated with shear layer/acoustic interactions in either the vaporization chamber or the aft-end mixing chamber of the motor ${ }^{6}$.

Instabilities associated with the (possibly) time-dependent nature of the boundary layer leading edge position (flameholder effects)?

\section{PRELIMIMARY ITSTABTITTY AMPLYSIS}

Low frequency, or so-called "non-acopstic" instabilities have been observed in a number of hybrid motor tests $2,3,6,8,9,10$. As the name implies, hybrid motors share common characteristics with both liquid propellant and solid propellant rocket motors. On the other hand, hybrids possess certain unique characteristics. For instance, the ability to throttle hybrids over a wide range 
of operating conditions, coupled with time-varying internal geometry (fuel grain configuration), increases the probability of encountering conditions favorable to the onset of instability ${ }^{3}$. In solid propellant motors the combustion process is assumed to be localized in a zone close to the propellant surface. The combustion is then treated as a boundary condition for the fluid dynamics problem, greatly simplifying a stability analysis by isolating chemical reactions from the cavity analysis. The combustion dynamics problem is then defined as the response of a thin combustion zone at the cavity wall to an imposed flow environment. In liquid propellant motors, on the other hand, the flow and combustion problems are coupled throughout the entire volume of the combustor.

Hybrid motor combustion typically occurs in a thin flame sheet located within a boundary layer located near the surface of the fuel grain. Fuel is partly decomposed and gasified at the solid surface by heat transfer and diffuses outward towards the flame zone. At the same time, oxidizer diffuses inward towards the flame zone through the boundary layer. At a point where the oxidizer-to-fuel concentration (O/F ratio) is slightly on the fuel-rich side of stoichiometric, combustion occurs in a zone whose thickness is about $10 \%$ of the boundary layer thickness. Some hybrid motors also inject oxidizer into a cavity located downstream of the solid fuel grain, so that a secondary combustion process occurs in this region. Hybrid motors can then be expected to exhibit the combustion characteristics of both solid and liquid rocket motors.

\section{Hrbrid Yotor I* Instability Analysis}

The approach taken in the present analysis is to develop a modified $L^{*}$ instability theory which accounts for the relationship between pressure and $0 / F$ ratio in the motor. The analysis proceeds along the lines of that first suggested by Beckstead and Price for solid propellant motors, with the following simplifying assumptions:

- The combustion process is assumed to be localized in a zone close to the propellant surface.

- The analysis is for one-dimensional flow of a calorically perfect gas. Temperature, molecular weight, and pressure are spacially uniform throughout the combustion volume. Molecular weight is either not oscillating or has a large oscillation period relative to the flow residence time (quasisteady).

- The nozzle discharge is quasisteady, with inlet stagnation conditions approximated by static conditions.

- All flow variables (except the oxidizer mass flow rate) can be expressed as the sum of a mean (constant) term and a time-dependent term

- The oxidizer mass flow is constant.

The continuity equation applied to the thrust chamber takes the form

$$
\frac{\tau_{c}}{n} \frac{d(p / \bar{p})}{d t}+\frac{p}{\bar{P}}=\frac{1}{1+\bar{\alpha}}+\frac{\bar{\alpha}}{1+\bar{\alpha}} \frac{\alpha}{\bar{\alpha}}
$$

where $p$ is pressure, $t$ is time, $(-)$ denotes a mean value, $\tau_{c}$ is the so-called motor fill (or flush) time, defined as

$$
\tau_{c}=\frac{L^{*} C^{*}}{R T}
$$


and

$$
\alpha=\frac{\dot{m}_{f}}{\dot{m}_{o}}
$$

is the inverse of the $0 / F$ ratio, and $n=1$ or $\gamma$ depending on whether the core flow is considered to be isothermal or isentropic. In the limit $\bar{\alpha} \rightarrow \infty, \mathrm{Eq} \cdot(1)$ degenerates to the solid rocket motor relations of Reference 11 . Now let

$$
\begin{gathered}
\frac{p}{\bar{p}}=1+\frac{p_{0}}{\overline{\bar{p}}} e^{\beta t} \cos (\beta t) \\
\frac{\alpha}{\bar{\alpha}}=1+\frac{\alpha_{0}}{\bar{\alpha}} e^{\beta t} \cos (\omega t+\tau)
\end{gathered}
$$

where $\beta$ is a growth rate constant for oscillations and $\tau$ is the lead time of the fuel-oxidizer ratio relative to the pressure. One can define a response function
as

$$
\psi \equiv \frac{\frac{\alpha_{0}}{\bar{\alpha}}}{\frac{p_{0}}{\bar{p}}}
$$

where $\alpha_{0}$ and $p_{0}$ are oscillation amplitudes of the fuel-oxidizer ratio and pressure at some (arbitrary) time $t$, respectively. Combining Eqs.(1)-(5) and solving yields two conditions (expressed in terms of $0 / F$ ratio):

$$
\begin{gathered}
\Psi \frac{1}{1+\bar{O} F} \cos (\omega \tau)=1+\beta \frac{\tau_{c}}{n} \\
\Psi \frac{1}{1+\overline{O / F}} \sin (\omega \tau)=\omega \frac{\tau}{n}\left(\frac{\tau_{c}}{\tau}\right)
\end{gathered}
$$

Equations (7) and (8) represent a set of 1-D stability oscillator equations for hybrid rocket motors. As such, they describe the dynamic behavior of a hybrid motor in terms of the oscillation variables $(\omega, \beta)$ as functions of the combustion dynamics $(\Psi, \tau)$, the mean $O / F$ ratio $(\overline{O / F})$, and the motor fill time ( $\left.\tau_{c}\right)$. The motor fill time is, in turn, dependent on $L^{*}$.

\section{Stability Irends}

If one considers the interval $-\pi<\omega \pi<$ for the combustion (i.e., fueloxidizer ratio) phase angle, one can quickly show that 
i.e., combustion oscillations lead pressure oscillations for all values of $\overline{0 / F}$, for either isothermal or isentropic core flow. Considering that the stability limit is defined by the condition $\beta=0$, one can show that the oscillator equations impose a minimum value constraint on the response function $\Psi_{\text {, and that }}$ this value is a function of the (mean) motor o/F ratio. This constraint is simply stated as

$$
\Psi_{0} \geq 1+\overline{O / F}
$$
where the subscript "o" denotes conditions at the motor stability limit. Thus, stability) as a solid propellant motor with the response function modified to account for O/F ratio. Finally, the expected pressure oscillation frequency can be estimated as

$$
f=\frac{n}{2 \pi \tau_{c}} \arccos \left(\frac{1}{\psi_{0}}\right)=f\left(\tau_{c}, \overline{O / F}\right)
$$

so that the expected oscillation frequency depends directly on the system fill time $\tau_{c}\left(\right.$ or $L^{*}$ ) and the average $0 / F$ ratio.

\section{Sample Results}

Guthrie and Wol $f^{3}$ reported non-acoustic instabilities in a series of motors, ranging in size from 10,000 lbs thrust to 75,000 lbs thrust, which had been developed and tested by the American Rocket company (AMROC). They then applied solid rocket motor instability theory and compared its predictions with their experimental data. In their work, they attempted to correlate data from "several solid fuels", and concluded that $\Psi=1.3$. According to Eq.(10) of the hybrid motor theory developed here, the correct value is $\Psi_{0}=3.09(i . e ., \overline{0} F \sim 2.09)$. A comparison of predicted vs. Observed oscillation frequencies is shown in Figure 1. Predicted frequencies are obtained using values of combustor fill time reported in Reference 3 . It can be seen that the hybrid motor theory provides excellent agreement with the observed values of oscillation frequency.

\section{SUMPRY IID COMGLUSIONS}

The hybrid stability theory presented here is a 1-D model derived along the same lines as for solid rocket motors, with account taken for a separate oxidizer mass flow. It represents a first step towards the understanding of non-acoustic oscillations in hybrid rocket motors. Since it is fundamental in nature, it necessarily employs a number of simplifying assumptions which restrict the applicability and adaptability of the model in more complex situations, and limit the type of information which the model can provide. Based upon the limited data presented here, the model can predict, to a reasonable degree, the frequency of non-acoustic oscillations in simple hybrid motor systems. It must be noted that the present theory cannot predict whether or not a given system will oscillate, nor can it suggest what measures should be undertaken to reduce or eliminate oscillations. 


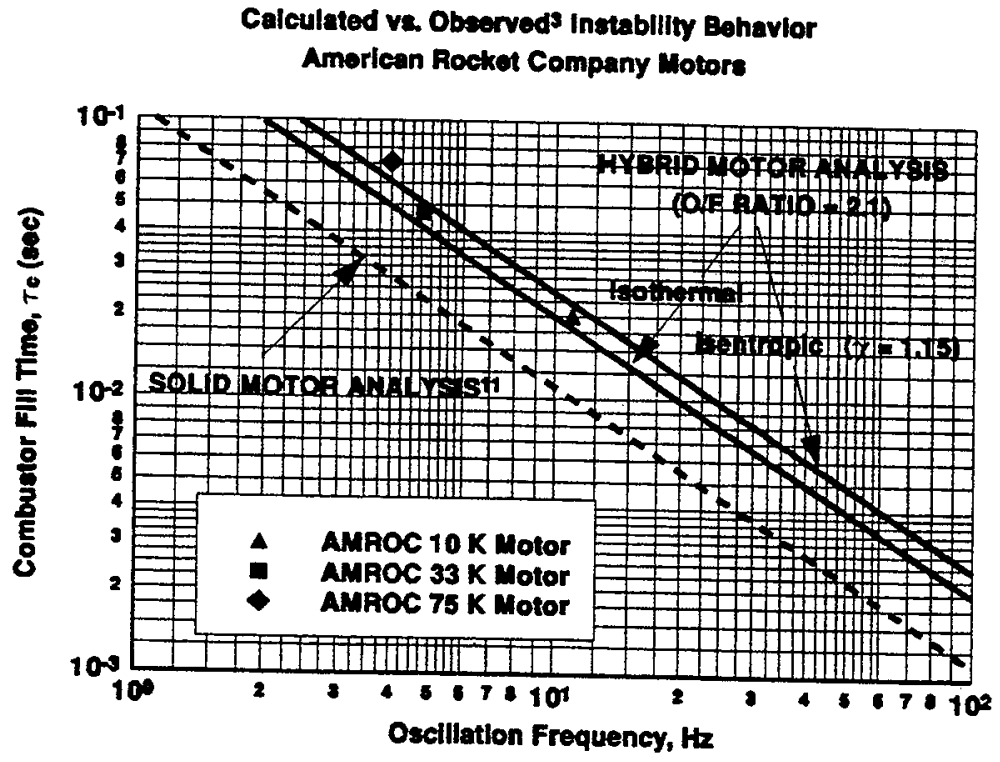

Fig. 1: Predicted vs. Observed Oscillation Frequency, AMROC Motors

\section{RBFERETICBS}

'G.P. Sutton, Rocket Propulsion Elements, 6th ed., Wiley and Sons, Inc., 1992. ${ }^{2}$ C.E. Wooldridge and G.A. Markman, "Combustion Instability and the Role of Chemical Kinetics in Hybrid Combustion," AIAA Paper No. 68-498, June 1968.

${ }^{3}$ D.M. Guthrie and R.S. Wolf, "Non-Acoustic Combustion Instability in Bybrid Rocket Motors," Report by American Rocket Company, Camarillo, CA, 1990. 4.M. Helmy, "Chronicle Review of the Hybrid Rocket Combustion," AIAA Paper No.
94-2881, June 1994. ${ }^{5}$.J. Muzzy, "Applied Hybrid Combustion Theory," AIAA Paper No. 72-1143, Nov.
1972.

6B. Greiner and R.A. Frederick, Jr., "Experimental Investigation of Labscale Hybrid Instability," AIAA Paper No. 94-2878, June 1994.

7 Personal Communication, Dr. Ronn Carpenter, Thiokol Corporation, June 1994.

\&.D. Strand, M.D. Jones, and R.L. Ray, "Characterization of Hybrid Rocket Internal Heat Flux and HTPB Fuel Pyrolysis," AIAA Paper No. 94-2876, 1994.

'B. Goldberg and J. Cook, "Preliminary Results of NASA/Industry Hybrid Propulsion Program," AIAA Paper No. 92-3299, 1992.

${ }^{10}$ M.H. Lee, C.F. Schafer, G.A. Robertson, D. Straub, R.H. Eskridge, and C.C. Dobson, "Pressure Oscillations in a Laboratory Scale Hybrid Motor," Conference Center, Buntsville, "1M.W. Beckstead and E.W. Price, "Nonacoustic Combustor Instability," AIAA
Journal, Vol. 5, No. 11, Nov. 1967. 